\title{
Jan RADIMSKÝ
}

Université de Bohême du Sud, České Budějovice

\section{COMPOSITION INDIGÈNE ET CONFIXATION : DEUX PROCÉDÉS CONCURRENTS EN ITALIEN CONTEMPORAIN}

\section{Introduction}

L'existence de deux structures de composition distinctes dans les langues romanes, dont l'une est dite «populaire » et l'autre « savante », est un fait reconnu par la plupart les romanistes, bien qu'en ce qui concerne les dénominations, le consensus soit loin d'être atteint. Les composés populaires sont parfois nommés « indigènes » ou «composés » tout court; par contre, leurs homologues savants, les confixés, reçoivent des étiquettes bien plus variées - en italien, par exemple, composti dotti, composti con elementi greco-latini, composti con elementi colti, composti con elementi scientifici, composti con prefissoidi e suffissoidi, composti con semiparole, etc. Si nous conservons la dénomination «confixé »d'André Martinet $^{2}$ pour un composé savant, c'est qu'elle est courte, elle permet de dénommer à la fois l'élément de la fusion («confixe») et le résultat de cette dernière («confixé ») et que finalement, elle était clairement définie par son auteur dans la perspective du structuralisme fonctionnel. ${ }^{3}$ Mais il n'en reste pas moins que dans notre conception, la confixation fait partie de la formation des mots par composition.

Les différences qui opposent les deux procédés de formation de composés se situent sur plusieurs plans linguistiques, mais dans la plupart des définitions, on insiste sur l'étymologie des éléments composants : les composés indigènes sont formés par la fusion des lexèmes populaires (finecorsa < fine, corsa), tandis que les confixés sont formés par les éléments savants empruntés au grec ou au latin (dermatoscopio < dermato /gr./, skópion /gr./). ${ }^{4}$ Cependant, l'évolution récente du lexique italien semble relativiser la distinction entre ces deux procédés, comme en témoigne l'apparition des composés difficilement classifiables dans les dernières

\footnotetext{
${ }^{2}$ Le terme italen «confisso », introduit en linguistique italienne en 1988 avec la traduction du livre Syntaxe générale de Martinet, a connu un succès notamment grâce au Grande dizionario italiano dell'uso de Tullio DE MAURo (1999). Toutefois, T. de Mauro désigne par le terme «confisso » pratiquement tous les éléments de composition dotés d'un sens plein et ayant formé au moins trois composés du dictionnaire (De MAURO, 1999 : 23), tandis que pour André MARTinet (1979 : 243244), un « confixe » est uniquement un élément de composition savant. Ainsi, les éléments copri- de coprifuoco ou porta- de portabagagli, considérés habituelement comme éléments verbaux d'un composé indigène du type $\mathrm{V}+\mathrm{N}$, figurent en tant que « confisso » parmi les entrées du GRADIT.

${ }^{3}$ Selon André MARTINET (1979 : 243-244), les propriétés typiques des confixés sont les suivantes : il s'agit des synthèmes constitués de deux monèmes non libérables, ou confixes; les confixés sont construits sur des modèles grecs et latins ; le premier confixe du confixé détermine habituellement le second ; les confixes sont facilement isolables grâce au riche paradigme des confixés qu'ils créent.

${ }^{4}$ RADIMSKÝ, 2006 : 58-61.
} 
décennies, ${ }^{5}$ et nous ammène jusqu'à repenser en théorie la distinction entre le composé indigène et savant.

Dans la présente contribution, nous nous proposons d'abord de confronter les deux procédés de formation de composés sur plusieurs plans linguistiques, afin de les distinguer plus nettement, pour ensuite passer en revue leur concurrence effective dans la langue contemporaine sur de différents niveaux. Pour ce faire, nous nous appuierons sur l'analyse d'un corpus de 1390 composés et confixés italiens actuels. Le corpus de recherche, analysé en détail dans notre thèse (RADIMSKÝ, 2005 et RADIMSKÝ, 2006), est constitué des entrées du dictionnaire GRADIT $^{6}$; nous avons choisi les entrées marquées comme «composto » (composé), dont la première apparition n'est pas antérieure à l'année 1980 et qui en même temps n'est pas un emprunt non adapté à une langue étrangère.

\section{Confrontation de deux prototypes}

\subsection{Présentation des prototypes}

Pour que la confrontation du procédé de composition «indigène » et «savant» soit plus explicite, nous allons présenter un lexème prototypique de chaque groupe, pour ensuite dégager et comparer leurs traits caractéristiques. Considérons donc le composé indigène finecorsa ${ }^{7}$ et le confixé dermatoscopio. ${ }^{8}$

Dans le cas de dermatoscopio, nous constatons que :

1) Les deux constituants (confixes) sont productifs (rentables). En effet, le GRADIT répertorie 50 confixés sur dermato- ${ }^{9}$ et 250 confixés sur -scopio. ${ }^{10}$

2) Les deux constituants sont dotés d'une signification référentielle correspondant à un adjectif (dermico - relatif à la peau) ou à un substantif (strumento usato per osservazioni - instrument servant à observer quelque chose).

\footnotetext{
${ }^{5}$ Cf. par exemple Pavao TeKavČić (1980 : 164-165). Claudio IACOBINI et Anna Thornton (1992: 28) introduisent une catégorie baptisée "formazioni di categoria indecidibile tra prefissazione e composizione » qui croît de $0 \%$ à $9,9 \%$ entre les parties de leur échantillon représentant respectivement le début et la fin du $20^{\mathrm{e}}$ siècle.

${ }^{6}$ De Mauro, Tullio (ideato e diretto da) : Grande dizionario italiano dell'uso, CD-ROM. Torino, Utet, 1999.

${ }^{7}$ Substantif masculin invariable, attesté pour la première fois en 1983 selon le GRADIT.

${ }^{8}$ Substantif masculin, attesté pour la première fois en 1995 selon le GRADIT.

${ }^{9}$ P. ex. dermatologia, dermatomicosi, dermatoplastica, dermatotropo, etc.

${ }^{10}$ P. ex. aeroscopio, artroscopio, broncoscopio, cardioscopio, etc.
} 
3) Les deux constituants ne sont pas libres (c'est à dire autonomes) : les formes *dermato- ni * -scopio ne sont pas attestées. Cependant, l'un des constituants admet la combinaison avec un affixe, sans recours à une base libre (autonome) : cf. dermatico de structure confixe+suffixe. Ainsi, il peut se comporter en base autonome.

4) Il s'agit d'une formation endocentrique comportant la tête à droite.

5) Les deux constituants ont une forme savante (venant directement du grec en l'occurrence).

Dans le cas de finecorsa, nous constatons par contre que :

1) Les deux constituants ne sont que peu productifs. En effet, le dictionnaire GRADIT répertorie seulement 4 composés sur fine- ${ }^{11}$ et 4 composés sur corsa. $^{12}$

2) Les deux constituants sont dotés d'une signification référentielle.

3) Les deux constituants sont libres, donc attestés comme lexèmes autonomes.

4) Il peut s'agir soit d'une formation endocentrique comportant la tête à gauche (interprétation la fine della corsa - fin du trajet), soit d'une formation exocentrique (interprétation dispositivo in grado di arrestare la corsa dispositif servant à freiner).

5) Les constituants n'ont pas une forme savante.

Voici un tableau récapitulatif des différentes propriétés :

\begin{tabular}{|l|l|l|}
\hline & Confixé & Composé indigène \\
\hline Productivité & grande & petite \\
\hline Signification référentielle des constituants & oui & oui \\
\hline Les constituants sont autonomes & non & oui \\
\hline Structure interne & $\begin{array}{l}\text { endocentrique, tête à } \\
\text { droite }\end{array}$ & $\begin{array}{l}\text { - endocentrique, tête à } \\
\text { gauche } \\
\text { - exocentrique }\end{array}$ \\
\hline Forme savante des constituants & oui & non \\
\hline
\end{tabular}

Suite à ce bref examen nous pouvons constater que les deux prototypes partagent seulement la propriété « signification référentielle des constituants » (cf. point 2). Examinons maintenant les autres propriétés, afin de trouver celle(s) qui serviraient le mieux à distinguer les deux procédés.

\subsection{Un confixé est-il toujours « savant »?}

Le caractère «savant» d'un confixé englobe en réalité deux propriétés distinctes parmi celles que nous venons de voir : il concerne non seulement la forme des éléments composants (confixes), mais également leur ordre dans le

\footnotetext{
${ }^{11}$ A savoir: finemillenio, finenovecentesco, finebianco, finegranulante.

${ }^{12}$ A savoir: autocorsa, cinocorsa, idrocorsa; cependant, les trois comportent en réalité un confixe responsable de la structure déterminant-déterminé. C'est pourquoi nous les considérons comme confixés.
} 
confixé (ordre déterminant - déterminé, typique pour les confixés). Concentronsnous maintenant uniquement sur la forme des éléments.

Du point de vue formel, les confixes savants ont été empruntés relativement tard aux langues classiques et n'ont donc pas subi d'altérations phonétiques importantes. Ainsi, la base latine aqua donne en français le lexème indigène eau, mais aussi le premier élément du composé acquaculture, de même que ostrea donne huître et ostréiculture, etc. Cependant, Louis Guilbert ${ }^{13}$ note, par exemple, que la distinction entre une forme savante et populaire ne doit pas être toujours aussi évidente, car au lexème latin mamilla s'associent en français la base mamelle et le confixé mamelliforme, les deux comportant le changement $i>e$.

Pour la langue italienne, le même problème se rencontre bien plus fréquemment, car les changements de forme entre le latin classique et l'italien moderne sont moins radicaux que dans le cas du français et par conséquent, les formes savantes et indigènes sont plus difficiles à distinguer. ${ }^{14}$ Après avoir consulté les dictionnaires de langue courants, nous constatons que les confixes «savants» subissent également certains changements propres aux mots indigènes; les altérations en questions sont répertoriées à titre d'exemple en trois catégories dans les tableaux suivants :

a) gémination d'une consonne :

aktînos > attino- $($ variante actino- possible $)$
omnis $>$ onni- $($ variante omni rare $)$
nyktós $>$ nitto- $($ variantes nicto-, nicti-, nitti- $)$
spectru $(m)>$ spettro $>$ spettro-
elektron $>$ electru $(m)>$ elettro / elettro-

b) altération $u>o$ (qui, à la fin du confixe, ne peut pas s'expliquer seulement par un réajustement dû à la composition, car l'altération est présente non seulement dans le confixe, mais également dans le lexème simple homonyme du confixe; le lexème simple, selon le dictionnaire, aurait été en usage avant le confixe) :

$$
\begin{aligned}
& \text { elektron }>\text { electru }(m)>\text { elettro } / \text { elettro- } \\
& \operatorname{servu}(m)>\text { servo }^{\prime}>\text { servo- }^{15} \\
& \text { flavu }(m)>\text { flavo }^{-} \text {flavo- }^{16} \\
& \operatorname{sexu}(m)>\operatorname{sesso} / \text { sesso- }^{-}
\end{aligned}
$$

\footnotetext{
${ }^{13}$ Cité d'après Jan KorTAS (2003 : 374).

${ }^{14}$ Edouard BouRCIEZ (1946) montre que l'évolution du système phonétique français par rapport au latin était bien plus radicale que celle de l'italien. Ceci concerne notamment le système vocalique (cf. BOURCIEZ, 1946 : 289-301, 480-485), mais également le système consonantique (cf. BourCIEZ, $1946: 301-313,485-494)$.

${ }^{15}$ Selon le GRADIT, le confixe servo- vient du confixe français ayant la même forme.

${ }^{16}$ Dans ce cas, la présence du groupe $f l$ (non palatalisé en fi) suffit à montrer le caractère savant de l'élément en question.
} 
$\operatorname{vasu}(m)>$ vaso $>$ vaso-

cultura $(m)>$ coltura $>$-coltura (la variante cultura est rare et vieillie)

c) diphtongaison $e>i e$ :

$\operatorname{seru}(m)>$ siero $>$ siero-

(la variante sero-, citée par le ZINGARELLI mais pas par le GRADIT, est probablement une monophtongaison récente due à l'influence des langues étrangères)

En effet, vu la similitude des formes classiques et modernes en italien, une seule altération phonétique suffit dans la plupart des cas à obscurcir la distinction entre les deux formes.

D'autre part, nous avons mis en évidence lors de nos recherches antérieures ${ }^{17}$ un nombre assez élevé d'éléments de composition qui se comportent, en synchronie, comme des confixes savants, mais qui ne viennent pas des langues classiques. Ces éléments peuvent être classés en trois groupes suivant leur étymologie (néoconfixes de sens, néoconfixes de forme et néoconfixes empruntés), comme le montre le tableau suivant :

\begin{tabular}{|c|c|c|c|c|}
\hline Type & $\begin{array}{c}\text { Confixe } \\
\text { classique }\end{array}$ & Lexème source & $\begin{array}{c}\text { Confixe } \\
\text { moderne }\end{array}$ & Exemple du confixé \\
\hline $\begin{array}{c}\text { Néoconfixes de sens } \\
\text { (à partir d'un confixe } \\
\text { classique) }\end{array}$ & auto- 1 > & $\underline{\text { automobile }>}$ & auto- 2 & $\begin{array}{c}\text { autodemolitore } \\
\text { autoconcessionario }\end{array}$ \\
\hline $\begin{array}{c}\text { Néoconfixes de forme } \\
\text { (à partir d'un mot italien) }\end{array}$ & --- & $\underline{\text { aviazione > }}$ & avio- & aviosuperficie $^{18}$ \\
\hline $\begin{array}{c}\text { Néoconfixes empruntés } \\
\text { (à partir d'un mot ou } \\
\text { confixe étranger) }\end{array}$ & --- & $\underline{\text { laser }}$ & laser- & laserchirurgia $^{-}$ \\
\hline
\end{tabular}

Ceci dit, force est de constater que d'une part, les confixes savants ne doivent pas toujours revêtir une forme savante, et d'autre part, il existe des confixes qui malgré leur origine non-savante, partagent en synchronie les propriétés de leurs homologues savants. Nous pouvons donc conclure en disant que le caractère savant est une propriété courante des confixes, mais non leur caractéristique principale. De plus, il s'agit d'une propriété de l'ordre diachronique qui, pour être pertinente en synchronie, aurait dû correspondre systématiquement à une autre propriété synchronique, ce qui ne semble pas être le cas. ${ }^{19}$

\footnotetext{
${ }^{17}$ RADIMSKÝ 2004, RADIMSKÝ 2006. Cf. également DARDANO (1994 : 422).

${ }^{18}$ L'expression désigne un type de piste de décollage.

19 Danielle CoRbin (1987 : 94-95) arrive à la même conclusion en disant : "Il ressort de ce qui précède qu'il n'est pas prouvé que le trait $[ \pm$ savant], référé à sa filiation historique, ait une quelconque pertinence dans une morphologie synchronique. [...] On rejettera donc de la présente théorie toute référence à un trait [ \pm savant], inadapté à une morphologie synchronique, sans pour autant refuser toute information d'origine historique».
} 


\subsection{La productivité}

Nous avons évoqué plus haut (cf. la section 2.1.) que si les confixes forment de riches paradigmes de confixés, les éléments de composition indigène donnent naissance à des paradigmes bien plus modestes. Les deux tableaux suivants illustrent le phénomène en indiquant le nombre de composés créés à partir d'une même base selon le dictionnaire GRADIT ; le premier tableau se rapporte aux bases confixales, tandis que le second rassemble les bases indigènes les plus productives (i.e. les bases verbales).

\begin{tabular}{|l|l|}
\hline Base confixale & Nombre des confixés dans le GRADIT \\
\hline auto & 606 \\
\hline micro & 529 \\
\hline poli & 526 \\
\hline pseudo & 490 \\
\hline mono & 456 \\
\hline neo & 405 \\
\hline radio & 357 \\
\hline foto & 268 \\
\hline tele & 244 \\
\hline bio & 189 \\
\hline multi & 162 \\
\hline pluri & 142 \\
\hline psico & 141 \\
\hline video & 85 \\
\hline
\end{tabular}

\begin{tabular}{|l|l|}
\hline Base indigène & Nombre des composés dans le GRADIT \\
\hline portare & 296 \\
\hline coprire & 64 \\
\hline lavare & 44 \\
\hline salvare & 30 \\
\hline
\end{tabular}

Du point de vue quantitatif, il y a une nette différence, mais il s'agit bien d'une différence scalaire, pas d'une différence vectorielle. De même que dans le cas précédent, nous pouvons conclure que la haute productivité des bases confixales par rapport aux bases indigènes est l'une des caractéristiques des deux procédés de composition, mais pas un critère qui soit apte à les différencier en tant que paradigmes.

\subsection{L'autonomie des éléments}

En termes de prototypes, un confixe pourrait être décrit comme généralement non autonome, tandis qu'un élément de composition indigène est (en italien) typiquement un lexème autonome. C'est ce que constate également André MARTINET (1979 : 243) pour le français, en ajoutant toutefois que «bien qu'ils ne soient pas libérables, les monèmes conjoints dans le confixé sont aisément identifiés du fait de leur présence dans de nombreux synthèmes de même modèle. Par ex. : -vore dans frugivore, omnivore, insectivore. » Cette propriété, appellée « autonomie de fonctionnement » par Jean PEYTARD (1975: 86) est probablement 
liée au fait que de nombreux confixes sont désormais susceptibles d'emplois autonomes. Dans le cas des confixes savants, l'emploi autonome peut être possible sans changement de signification (p. ex. mania, fobia, farmaco, spettro, pausa,

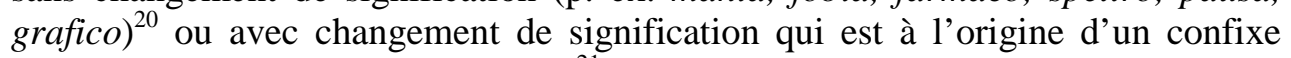
nouveau (p. ex. auto, euro, radio). ${ }^{21}$ Dans le cas des confixes non savants en italien, nous estimons que le rapport des éléments autonomes atteint même environ $50 \% .^{22}$

L'autonomie d'un élément lexical est donc fonction de temps, et l'évolution du composé et celle de ces éléments est en quelque sorte indépendante, aussi bien dans les confixés que dans les composés indigènes. Ainsi, par exemple le second élément du composé français porc-épic n'existe plus aujourd'hui. Les deux éléments du composé contredire existent encore, mais la forme fléchie de la $2^{\mathrm{e}}$ personne de l'indicatif au pluriel (vous contredisez vs. vous dites) témoigne du fait qu'il est impossible de "générer » simplement ce composé à partir des règles et des éléments contemporains.

Considérant l'instabilité relative de l'autonomie des éléments lexicaux, nous croyons qu'il s'agit là encore d'un trait caractéristique, mais non essentiel, de la distinction de nos deux paradigmes - que ce problème de l'autonomie soit étudié dans la perspective de la linguistique fonctionnelle (qui cherche plutôt à savoir si les éléments étaient autonomes au moment de la fusion du composé) ou bien dans celle de la linguistique générative (qui se demande si les éléments existent comme autonomes au moment où le composé est utilisé). ${ }^{23}$

\subsection{L'ordre des éléments dans le composé}

Comme nous l'avons évoqué dans la section 2.1., un composé indigène est soit exocentrique (en italien, c'est typiquement le cas des composés $\mathrm{V}+\mathrm{N}$ ), soit endocentrique, comportant la tête à gauche, c'est à dire que les éléments suivent l'ordre déterminé-déterminant. Par contre, le confixé serait endocentrique tout en ayant la tête à droite, c'est à dire que les éléments suivent l'ordre déterminantdéterminé. En effet, l'ordre déterminant-déterminé reflète l'ordre usuel dans les composés grecs et latins, sans qu'il soit pour autant un miroir parfait du modèle classique, comme l'avait brillamment démontré Emile Benveniste en étudiant le cas de microbe. ${ }^{24}$ Par conséquent, le modèle déterminant-déterminé peut être considéré comme classique et moderne à la fois, sa modernité étant accentuée par

\footnotetext{
${ }^{20}$ Pour mania e fobia il y a eu d'abord le statut lié suivi de l'autonomisation, les autres sont attestés comme autonomes avant leur emploi confixal.

${ }^{21}$ Auto-1 dans l'acception «soi-même», auto dans l'acception «automobile»; euro- 1 dans l'acception «européen », euro comme «monnaie unique »; radio-1 dans l'acception « radiation», radio comme «appareil radiophonique ». Les nouveaux lexèmes autonomes fonctionnent également comme confixes, par exemple dans : autodemolitore, eurozona, radiodrammaturgia.

${ }_{22}$ Plus exactement, nous avons mis en évidence 93 néoconfixes dont 47 autonomes et 46 non autonomes. Cf. RADIMSKÝ (2006: 132).

${ }^{23}$ Cf. RADIMSKÝ (2006 : 30-31).

${ }^{24}$ Benveniste, 1974.
} 
le fait qu'il se montre de plus en plus apte à s'appliquer aux éléments de composition non savants.

En ce qui concerne l'aptitude de ce critère à établir la différence entre les paradigmes indigène et confixal, nous observons qu'il permet d'éviter les inconvénients rencontrés lors de l'analyse des critères mentionnés précédemment :

a) Il s'agit d'un critère purement morphosyntaxique, donc pertinent en linguistique synchronique, et facilement identifiable.

b) Il n'établit pas une différence scalaire, décrivant un différent degré d'une même propriété, mais vectorielle (c'est à dire dans quelle direction il faut analyser le composé) qui est binaire (ordre déterminant-déterminé vs. toute autre structure).

c) A la différence du critère de l'autonomie, il est indépendant de l'évolution des éléments de composition.

C'est pourquoi nous le considérons comme essentiel dans la distinction entre la composition indigène et la confixation en italien.

\section{Concurrence des deux paradigmes dans la langue contemporaine}

\subsection{Concurrence au niveau de la productivité}

D'un point de vue strictement quantitatif, les confixés prévalent nettement sur les composés indigènes dans notre corpus de recherche, comme le montre le graphique suivant ( $1 \%$ correspond à 13,9 lexèmes en chiffres absolus) :

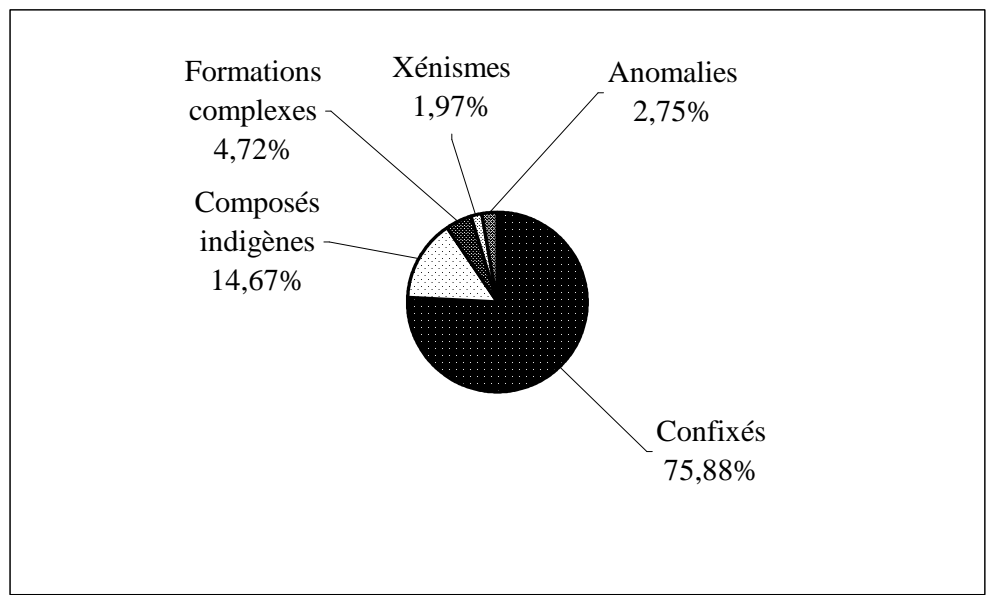

La prépondérance des confixés peut s'expliquer par le fait qu'ils s'assemblent souvent en riches paradigmes crés à partir d'une même unité confixale, comme nous l'avons vu dans la section 2.3. Facilement compréhensibles grâce à leur structure régulière (déterminant-déterminé) et grâce au recours aux unités confixales claires, les confixés modernes se situent au centre de la créativité lexicale par composition. 


\subsection{Concurrence sur le plan sociolinguistique}

On considère généralement que les confixés relèvent surtout du domaine des langues de spécialité, d'où viennent également certaines de leurs dénominations (p. ex. « composti scientifici» chez Luca Serianni ${ }^{25}$ ). Certains auteurs admettent cependant, qu'au cours du $20^{\mathrm{e}}$ siècle, la vulgarisation des termes scientifiques entraine une extension progressive de la confixation à la langue commune (DARDANO, 1994 : 422). Les données rassemblées dans notre corpus permettent également d'illustrer ce clivage, car les auteurs du GRADIT ont assigné à chaque entrée une marque d'usage. ${ }^{26}$ Les deux tableaux suivants indiquent la répartition de ces marques pour chaque type d'unités, confixés et composés indigènes.

Marques de registre parmi les confixés

\begin{tabular}{|l|r|c|}
\hline \multicolumn{1}{|c|}{ Catégorie } & Nombre de lexèmes & Taux \\
\hline Termes spécialisés & 719 & $66,82 \%$ \\
\hline Vocabulaire commun & 281 & $26,12 \%$ \\
\hline Rares & 73 & $6,78 \%$ \\
\hline Sans indication & 3 & $0,28 \%$ \\
\hline Total: & $\mathbf{1 0 7 6}$ & $\mathbf{1 0 0 \%}$ \\
\hline
\end{tabular}

Marques de registre parmi les composés indigènes

\begin{tabular}{|l|r|r|}
\hline \multicolumn{1}{|c|}{ Catégorie } & Nombre de lexèmes & Taux \\
\hline Termes spécialisés & 67 & $32,21 \%$ \\
\hline Vocabulaire commun & 109 & $52,40 \%$ \\
\hline Rares & 31 & $14,90 \%$ \\
\hline Dialectaux & 1 & $0,48 \%$ \\
\hline Total: & $\mathbf{2 0 8}$ & $\mathbf{1 0 0 \%}$ \\
\hline
\end{tabular}

Il faut admettre que les termes spécialisés ont un taux deux fois plus élevé parmi les confixés (67\% contre $32 \%$ pour les composés indigènes). Toutefois, le vocabulaire commun représente dans le groupe des confixés un quart des lexèmes. Si l'on compare les chiffres absolus, on constate que dans le domaine du vocabulaire courant, le dictionnaire enregistre 109 composés indigènes nouveaux contre 281 confixés nouveaux.

Ceci nous amène à conclure que la confixation n'est pas un procédé uniquement propre aux langues de spécialité; la langue courante a même plus souvent recours à la confixation qu'à la composition indigène.

\footnotetext{
${ }^{25}$ SERIANNI, $2000: 465$.

${ }^{26}$ Les trois principales marques utilisées sont: « $\mathrm{CO} »($ mot courant), «TS » (terme spécialisé, souvent avec une indication supplémentaire précisant le domaine d'utilisation), «BU » (mot rare). Lorsque l'entrée était marquée « $\mathrm{CO}$ » et « TS » à la fois, nous avons pris en compte la marque $\ll \mathrm{CO} »$ dans ce calcul.
} 


\subsection{Concurrence au niveau morphosyntaxique}

Selon le critère de l'ordre des éléments constitutifs des confixés et des composés indigènes, les deux paradigmes étudiés devraient être parfaitement complémentaires (c'est à dire que les confixés auraient toujours la structure déterminant-déterminé, tandis que les composés indigènes auraient une structure différente). Toutefois, l'existence simultanée de trois faits, dont chacun paraît non pertinent à première vue, risque de déséquilibrer non seulement cette complémentarité, mais le principe même de la distinction entre les deux paradigmes. Les trois faits sont les suivants:

- parmi les confixés mis en évidence, la structure $\mathrm{C}+\mathrm{N}$ (confixe + nom) prévaut nettement, ${ }^{27}$

- parmi les composés indigènes, la structure $\mathrm{N}+\mathrm{N}$ est l'une des productives,

- de nombreux confixes (C) peuvent être autonomes (cf. 2.4.), le plus souvent en tant que substantifs $(\mathrm{N})$.

En effet, nous affirmons que la composition nominale (par exemple du type $\mathrm{N}+\mathrm{N}$ ) est fondamentalement différente par sa structure interne de le confixation (par exemple du type $\mathrm{C}+\mathrm{N}$ ), mais qu'en même temps, certains confixes sont susceptibles d'emploi substantival $(\mathrm{C}=\mathrm{N})$. Dans la réalité linguistique, cette contradiction se traduit par un problème dans l'interprétation. Si nous analysons par exemple le composé fotoarchivio en tant que formation indigène, nous obtenons la signification photographie d'archive ou photographie des archives; par contre, en analysant le même mot en tant que confixé, la signification devient archives de photographies - tout ceci étant dû au fait que le premier élément foto apparaît comme premier élément des confixés et comme un substantif autonome. Comment pourrait-on rémédier à ce problème ?

Rappelons que de nombreux grammairiens, dont par exemple Sergio SCALISE (1994 : 269-271), affirment que la distinction entre les confixés et les composés indigènes ${ }^{28}$ est basée sur une propriété spécifique (trait +/- savant) des éléments constituants. Autrement dit, certains éléments (savants) participent seulement à la formation des confixés, tandis que d'autres (non savants) servent à former uniquement les composés indigènes. Ainsi, le risque d'interférence morphosyntaxique n'existe pas et les deux procédés compositionnels contradictoires peuvent coexister en une seule langue. A notre avis, si la distinction entre les éléments savants et indigènes s'efface, l'équilibre du système n'en doit pas être bouleversé pour autant. Nous avançons par analogie l'hypothèse que certains éléments, appelés confixes, participent uniquement à la composition du type confixal (ordre déterminant-déterminé) et n'apparaissent jamais (sauf quelques cas exceptionnels) dans la composition indigène du type $\mathrm{N}+\mathrm{N}$.

Examinons sous cet aspect les mots «composés » du GRADIT, contenant l'élément foto.

\footnotetext{
${ }^{27}$ La structure $\mathrm{C}+\mathrm{N}$ a été repérée chez 529 lexèmes, soit $49 \%$ des confixés du corpus.

28 «Composti dotti » et « composti » tout court, dans la terminologie de cet auteur.
} 
L'élément «foto » en première position

\begin{tabular}{|l|l|l|}
\hline \multicolumn{1}{|c|}{ Lexème } & \multicolumn{1}{|c|}{ CM } & \multicolumn{1}{c|}{ Signification } \\
\hline fotobiografia & s f & biografia per immagini \\
\hline fotodisco & s m & $\begin{array}{l}\text { particolare caricatore costituito da un supporto circolare su } \\
\text { cui sono inseriti piccoli riquadri di pellicola, utilizzabile su } \\
\text { apposite macchine fotografiche | sistema di fotografia che } \\
\text { utilizza tali caricatori }\end{array}$ \\
\hline fotogiornalismo & s m & giornalismo realizzato attraverso servizi fotografici \\
\hline fotogiornalista & s mf & chi effettua servizi giornalistici completi di testi e fotografie \\
\hline fotolaboratorio & $\mathrm{s} \mathrm{m}$ & laboratorio fotografico \\
\hline fotolibro & $\mathrm{s} \mathrm{m}$ & libro fotografico che tratta spec. argomenti di attualità \\
\hline fotopiano & $\mathrm{s} \mathrm{m}$ & rappresentazione di un piano urbanistico attraverso fotopiante \\
\hline fotosafari & $\mathrm{s} \mathrm{m} \mathrm{inv}$ & safari fotografico \\
\hline fotoservizio & $\mathrm{s} \mathrm{m}$ & reportage fotografico \\
\hline fotostoria & $\mathrm{s} \mathrm{f}$ & racconto realizzato con una serie di immagini fotografiche \\
\hline fototelegramma & $\mathrm{s} \mathrm{m}$ & immagine trasmessa mediante un apparecchio fototelegrafico \\
\hline
\end{tabular}

L'élément «foto » en seconde position

\begin{tabular}{|l|l||l|}
\hline Lexème & CM & Signification \\
\hline laserfoto & s f inv & $\begin{array}{l}\text { fotografia presa o trasmessa con apparecchiature a raggi laser } \\
\text { tecnica relativa a tale tipo di fotografia }\end{array}$ \\
\hline pornofoto & s f inv & foto pornografica \\
\hline
\end{tabular}

En examinant les définitions il y a lieu de constater qu'en première position, foto est toujours déterminant, tandis qu'en seconde position il est toujours déterminé. Par conséquent, tous les composés énumérés sont des confixés ayant la structure $\mathrm{C}+\mathrm{N}$ (premier tableau) ou $\mathrm{C}+\mathrm{C}$ (second tableau), aucune structure indigène du type $\mathrm{N}+\mathrm{N}$ n'est repérée.

L'hypothèse que nous venons d'exposer a été vérifiée sur la totalité du corpus étudié, c'est à dire sur 1390 composés italiens datant des années 1980-1999, où les contre-exemples étaient exceptionnels. ${ }^{29}$ Cependant, une vérification complète devrait sans aucun doute prendre en considération également les composés plus anciens - du moins ceux créés depuis l'unification de l'Italie au $19^{\mathrm{e}}$ siècle. En effet, nous sommes également persuadés qu'elle ne pourra pas se passer d'une étude systématique des structures contenant un nom transposé en adjectif (dans les structures du type personaggio chiave, posizione chiave, etc., écrites en deux mots graphiques), car ces dernières peuvent revêtir la forme d'un confixé « devant le miroir, » comme il résulte des exemples suivants ${ }^{30}$ :

\footnotetext{
${ }^{29}$ P. ex. fototessera, discogay - cf. Jan RADIMSKÝ (2006 : 148-155) pour une analyse détaillée.

${ }^{30}$ Le phénomène a été constaté déjà par Bruno MigLioRINI (1963:34), mais à notre connaissance, aucune recherche ne s'en est systématiquement pas occupée.
} 


\begin{tabular}{|l|l|}
\hline Structure « N+N » & Structure « $\mathbf{C + N}$ » \\
Exemples du corpus CILTA & Entrées du GRADIT \\
\hline antenna radio & - \\
\hline canale radio & radiocanale \\
\hline frequenza radio & radiofrequenza \\
\hline onda radio & radioonda \\
\hline segnale radio & radiosegnale \\
\hline
\end{tabular}

Quoique ce dernier problème dépasse déjà le cadre de l'étude des composés que nous nous étions fixé, une description théorique de la composition des mots en italien ne pourra pas l'omettre, car ses conséquences sur le plan de la linguistique générale pourraient être importantes.

\section{Conclusion}

La concurrence de la composition indigène et savante en italien contemporain, nous l'avons montré, connaît des mutations profondes. Sur le plan théorique, nous proposons de remplacer les critères de distinction traditionnels entre les deux paradigmes, comme celui de l'autonomie et l'origine savante des éléments, par un critère unique - celui de la relation syntaxique entre les éléments. Pour les confixés, «l'autonomie de fonctionnement» des éléments s'avère un paramètre plus apte à l'analyse que leur « autonomie » tout court. Nous avons également vu que la confixation était devenue un procédé beaucoup plus rentable par rapport à la composition indigène, et ceci même dans la langue commune. Au niveau morphosyntaxique, la confixation crée de riches paradigmes à partir des éléments (confixes) qui, s'ils sont autonomes, ne participent pas à la composition indigène, ce qui est un moyen d'éviter la confusion dans l'interprétation du lexique composé.

\section{BIBLIOGRAPHIE}

Benveniste, Emile (1974), Formes nouvelles de la composition nominale. Problèmes de linguistique générale 2, Paris, Gallimard, p. 163-176.

BOURCIEZ, Edouard (1946), Elements de linguistique romane. Paris, Klincksieck.

CORBIN, Danielle (1987), Morphologie dérivationnelle et structuration du lexique Vol. 1. Tübingen, Niemeyer.

DARDANO, Maurizio (1994), Profilo dell'italiano contemporaneo. In : Serianni L., Trifone P. (a cura di), Storia della lingua italiana, vol. II, Torino, Einaudi.

IACOBINI, Claudio, \& THORNTON, Anna Maria (1992), Tendenze nella formazione delle parole nell'italiano del ventesimo secolo. Linee di tendenza dell'italiano contemporaneo, Atti del XXV Congresso, Roma, Bulzoni, p. 25-55.

MARTINET, André (1979), Grammaire fonctionnelle du français. Paris, Didier Crédif.

PEYTARD, Jean (1975), Recherches sur la préfixation en français contemporain. Thèse dactylographiée en 3 volumes, Honoré Champion, Paris. 
RADIMSKÝ, Jan (2004), Les nouveaux confixes en italien contemporain. In: Pešek O. (ed.), Opera romanica 5, Langue et société - Dynamique des usages, České Budějovice, Jihočeská univerzita, p. 151-157.

RADIMSKÝ, Jan (2005), Les composés italiens actuels. Thèse de doctorat, soutenue à la Faculté des lettrers, Université Charles, Prague.

RADIMSKÝ, Jan (2006), Les composés italiens actuels. Cellule de recherche en linguistique, Paris, 2006.

SERIANNI, Luca (2000), Italiano. Torino, Garzanti.

TEKAVČIĆ, Pavao (1980), Grammatica storica dell'italiano, vol. III., Il lessico. Bologna, Mulino.

\section{RIASSUNTO}

\section{Composizione popolare e confissazione: due procedimenti concorrenti nell'italiano contemporaneo}

Il fenomeno della composizione di parole nelle lingue romanze rappresenta principalmente due procedimenti diversi : la composizione popolare e la confissazione, chiamata anche composizione dotta. Tradizionalmente, i due procedimenti vengono distinti secondo una serie di criteri, tra cui l'origine dei costituenti del composto è il più importante. Però l'analisi di questi criteri ci mostra che i due procedimenti vanno distinti piuttosto secondo la loro struttura morfosintattica interna; in più, il criterio tradizionale dell'autonomia limitata dei confissi cede il passo al concetto dell'«autonomia di funzionamento », descritta già da Peytard.

Basandoci su una ricerca di 1390 composti e confissati italiani recentissimi, mettiamo a confronto i due procedimenti nella lingua contemporanea da tre punti di vista : la loro produttività, il registro d'uso delle apposite neoformazioni e la morfosintassi interna di questi ultimi. L'analisi morfosintattica ci permette di formulare l'ipotesi che i confissi, anche quelli di origine popolare, non fanno mai parte dei composti popolari. Questo fenomeno, insieme all'autonomia di funzionamento dei confissi, dovrebbe permettere ad un madrelingua italiano di interprettare correttamente composti e confissati sconosciuti. 